\title{
JND Analysis of Texture Roughness Perception using a Magnetic Levitation Haptic Device
}

\author{
Bertram Unger, Ralph Hollis and Roberta Klatzky \\ Robotics Institute \\ Carnegie Mellon University \\ 5000 Forbes Avenue, Pittsburgh PA, USA
}

\begin{abstract}
This paper describes the use of a magnetic levitation haptic device (MLHD) to study the psychophysics of texture roughness. Studies of texture roughness perception performed using real textures can be time consuming and expensive. By using a MLHD to simulate texture we are able to quickly and easily adjust texture parameters. A dithered textured surface composed of conical elements is simulated using a constraint surface algorithm. The constraint surface shape is defined by the geometry of the elements as well as the size and shape of the virtual probe. The spacing of the elements and the size of the probe can be varied continuously and in real time.

Just noticeable difference (JND) experiments were conducted over the parameters of probe radius and texture spacing. The JND of roughness was determined with respect to element spacing using unforced weighted up-down adaptive threshold estimation. JND's were found to vary for texture spacing and probe size. JND's for constant probe size decreased with increasing texture spacing to a minimum and then increased again. JND's for constant spacing increased as probe size increased. These results are consistent with a geometric model of probe-texture interaction.
\end{abstract}

\section{Introduction}

Texture is an important part of the way we haptically perceive the world. The roughness of a tabletop may help distinguish wood from marble and the texture of clothing can affect our perception of its quality.

How texture is perceived is still an open question. It has been shown that for the bare finger, spatial effects are important while vibratory effects play a role in fine texture discrimination $(<100 \mu \mathrm{m})$ [5, 11]. It has, however, been demonstrated that texture perception via a probe must be mediated by vibration [8]. How the nature of the vibratory stimulus matches a texture's perceptual characteristics is still unknown.

Studies have shown that the relationship of probe radius to texture element spacing has a significant effect on subjective roughness estimates $[9,10]$. In this study the probesize/texture-spacing relationship is examined using a just noticeable difference (JND) technique.

We wish to determine in particular whether the combined spacing/probe parameters that lead to the greatest subjective roughness also lead to the greatest discriminability. If so, the JND should be inversely related to the relative roughness magnitude. Moreover, the mechanical interactions that cause the two effects, one on intensity and one on discriminability, can potentially be determined.

Despite the importance of this issue, to the best of our knowledge, this has never been done before. Determining real world JND's is typically a difficult process. Subjects are repeatedly asked to discriminate between two objects as the difference between them is gradually reduced. Eventually a discrimination threshold is determined [14]. Since the way in which the texture-spacing/probe-size relationship affects roughness perception is being studied, it is necessary to produce a set of many textures with varied spacings as well as a set of probes with differing radii. For real textures this can be an expensive and exacting process, particularly if elements are small or tightly spaced. A haptic device can be used to quickly and easily render textures over a continuous range of spacings [12]. Previous measurements of amplitude thresholds for sinusoidal gratings in real and virtual environments demonstrate the validity and usefulness of a haptic device for JND studies [16].

In order to compare this study's findings with experiments using real textures, the haptic device employed will need to be able to produce frequencies similar to those thought to mediate roughness perception - from 10-250 $\mathrm{Hz}$ [5]. It should also be capable of rendering a hard surface with at least $0.8-1.0 \mathrm{~N} / \mathrm{mm}$ stiffness to prevent perceptual instability [2] and have a resolution capable of accurately 
rendering small elements $(0.5-3.5 \mathrm{~mm}$ for spacing and element diameters of $1.05 \mathrm{~mm}$ and heights of $400 \mu \mathrm{m}$ in a previous study [9]). The magnetic levitation haptic device (MLHD) used in these experiments meets or exceeds these requirements [1].

Exact representations of texture surface topology are required so stochastic algorithms [4, 15] are not useful. Rather a constraint-surface algorithm, similar to virtual proxy techniques $[13,17]$ is used to generate a $3 \mathrm{D}$ probe shape around a haptic interaction point (HIP). This allows representation of the interaction of a spherical probe with geometrically deterministic surface topology (see Fig. 1).

The constraint surface texture simulation is used to determine JND's for roughness perception using a MLHD. This study also examines how JND varies with respect to spacing and probe size and how this relates to existing models of texture roughness perception.

\section{Haptic Device Design}

Typical haptic devices have friction, backlash and inertia associated with their actuators and linkages. Their position bandwidths and resolutions are often insufficient to render the rapid vibratory motions associated with texture. The device chosen for this experiment is a 6-DOF magnetic levitation haptic device [1]. It has a position resolution of 5-10 $\mu \mathrm{m}$ allowing simulation of the small elements needed for texture simulation. A maximum stiffness of $25 \mathrm{~N} / \mathrm{mm}$ in translation and $50.0 \mathrm{Nm} / \mathrm{rad}$ in rotation is sufficient to render perceptually hard surfaces. The most significant limitation of the MLHD is its small workspace $( \pm 12 \mathrm{~mm}$ in translation, $\pm 7^{\circ}$ rotation about any axis) [1]. This limitation is actually an advantage for texture simulation, restricting the user's hand to small, writing-like motions.

Vibrations transmitted to the user's hand through the device's virtual probe are thought to affect roughness perception [8]. The MLHD must therefore be capable of accurately reproducing high frequency motion and requires a high position bandwidth. The MLHD has been modeled as a second order spring-damper system using measurements of the MLHD's damping and spring coefficients. These prove to be highly linear. The modeled frequency response has a $\pm 3 \mathrm{~dB}$ corner at approximately $120 \mathrm{~Hz}$ with a slow roll off. It should therefore be able to produce texture with appropriate vibrational components.

\section{Constraint Surface Texture Model}

The constraint surface model developed describes the motion of a spherical probe tip across a set of truncated conical elements. The starting point for this model is a static geometric model for similar surfaces proposed by Klatzky et al. [9].

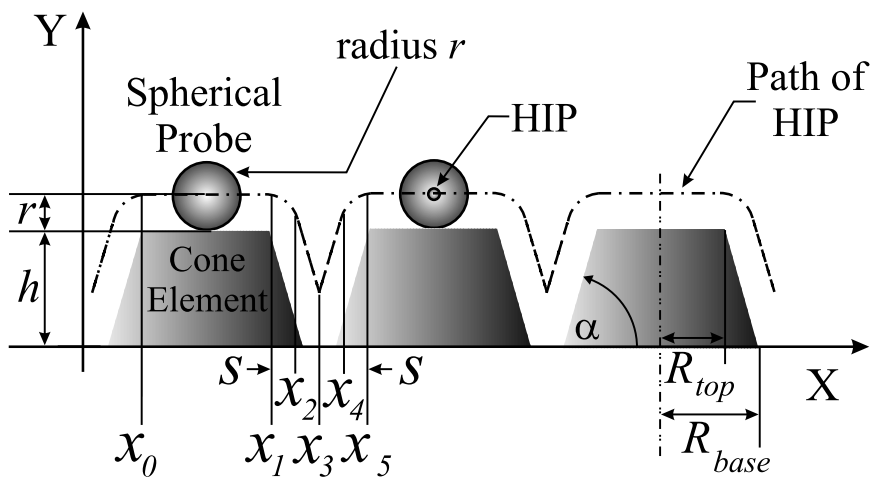

Figure 1. Cross-section of conical texture elements with measurements for probe path calculations.

If the HIP is considered to be the center of a spherical probe tip, it will need to be constrained to travel along the periodic surface seen in Fig. 1 as a dashed line. This constraint surface is generated by a piecewise-continuous set of functions defined by the interaction between the shape of the probe tip and the topology of the surface.

As seen in Fig. 1, the texture model uses a set of truncated cones. Each cone has a height $h$ and the sides of the cone rise with angle $\alpha$ to a plateau. A separation $s$ is designated as the distance between the lip of one plateau and the next. If a spherical probe of radius $r$ travels around a convex corner that has greater curvature than the sphere itself, it moves along an arc with radius $r$. Otherwise, it moves a distance $r$ from the surface and parallel to it. The exception to this is at $x_{3}$, the midpoint $(s / 2)$, where a discontinuity occurs if the sphere is too wide to reach the bottom of the groove between cones. If the sphere is able to reach the groove bottom, it will move parallel to the bottom until it contacts the next ascending slope.

For the following intervals from Fig. 1, the height of the sphere's center above the $x$-axis is defined by the equations:

$$
\begin{gathered}
x_{0} \leq x<x_{1}: y=h+r, \\
x_{1} \leq x<x_{2}: y=h+\sqrt{r^{2}-\left(x-x_{1}\right)^{2}}, \\
x_{2} \leq x<x_{3}: y=\tan (\alpha)\left(x_{2}-x\right)+y_{2}, \\
x_{3} \leq x<x_{4}: y=\tan (\alpha)\left(x-x_{3}\right)+y_{3}, \\
x_{4} \leq x<x_{5}: y=h+\sqrt{r^{2}-\left(x-x_{5}\right)^{2}},
\end{gathered}
$$

where $y_{2}$ and $y_{3}$ are the sphere height at $x_{2}$ and $x_{3}$ respectively.

To use this model to constrain the HIP to travel above a surface of cones as if it were a sphere, the model uses the interval in which the HIP's $x$ position lies to calculate the corresponding desired height, $y$.

This algorithm is modified in order to generate the constraint surface for a dithered set of cones. The center points 


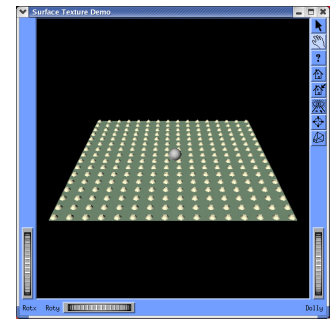

(a)

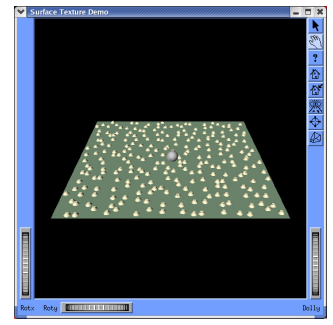

(b)
Figure 2. a.) Undithered cone texture with $1.0 \mathrm{~mm}$ radius probe on $2.0 \mathrm{~mm}$ cone spacing. b.) Cone texture with 1.0 $\mathrm{mm}$ radius probe and $2.0 \mathrm{~mm}$ cone spacing dithered by 0.8 $\mathrm{mm}$.

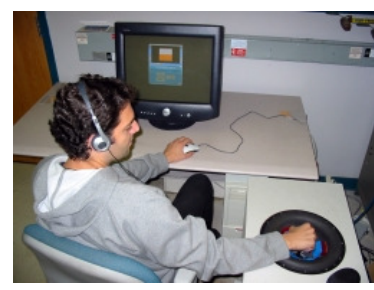

(a)

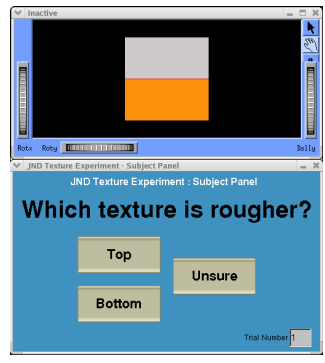

(b)
Figure 3. a.) Subject using the magnetic levitation haptic device during JND experiments. b.) JND experiment subject panel with selection buttons and visual active panel display.

was provided. Subjects listened to white noise using headphones to prevent auditory identification of textures.

\begin{tabular}{|c|c|c|c|c|c|}
\hline Probe Radius & \multicolumn{5}{|c|}{ Base Texture Spacing [mm] } \\
\cline { 2 - 6 }$[\mathrm{mm}]$ & BT1 & BT2 & BT3 & BT4 & BT5 \\
\hline 0.25 & 0.50 & 0.625 & 0.75 & 0.875 & 1.00 \\
0.50 & 0.50 & 0.65 & 0.80 & 0.95 & 1.10 \\
1.00 & 0.50 & 0.75 & 1.00 & 1.25 & 1.50 \\
1.50 & 0.50 & 0.875 & 1.25 & 1.625 & 2.00 \\
\hline
\end{tabular}

Table 1. Probe radius and base texture spacing used for JND experiments.

The experiment was divided into four blocks, presented to subjects randomly. In each block a spherical probe of set radius was used to examine five BT spacings (see Table 1). Subjects thus determined 20 JND's. Each experiment lasted approximately an hour.

A version of Kaernbach's unforced weighted up-down adaptive threshold estimation was used to rapidly determine the JND [7]. Using this technique subjects are asked to discriminate between a base and a comparison texture's roughness. If the subject chooses correctly, the difference $\delta$ between BT and CT spacing is reduced by stepsize $D 1$. In the case of an incorrect answer, $\delta$ is increased by a stepsize of $D 2$. An indeterminate answer also results in an increased difference but by a smaller increment $D 3 . D 1$ is chosen arbitrarily, based on empirically determined convergence times. $D 2$ and $D 3$ are chosen to achieve our desired performance rate $p$ at a threshold of $75 \%$ correct [7].

An initial $D 1$ of $10 \%$ of the initial $\delta$ was used and $D 2$ and $D 3$ were determined accordingly. As the experiment progresses the CT spacing should be observed to move to- 

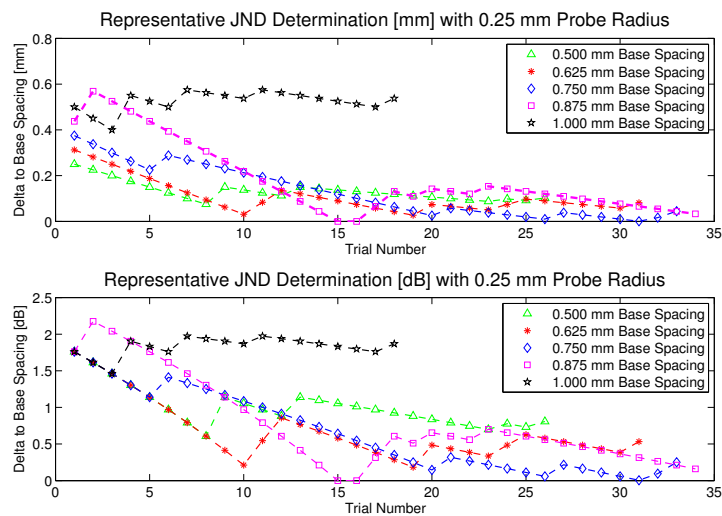

Figure 4. Determining JND using unforced weighted updown adaptive threshold estimation. JND determinations for five base texture spacings are shown.

wards the BT spacing until an equilibrium is reached (see Fig. 4). The number of direction reversals in the path of $\delta$ are then measured. At 2 and 4 reversals all stepsizes are reduced by a factor of 2 . Once eight reversals have occurred the JND is determined as the mean of all $\delta$ 's from the value just after the fourth reversal to the value of the eighth reversal

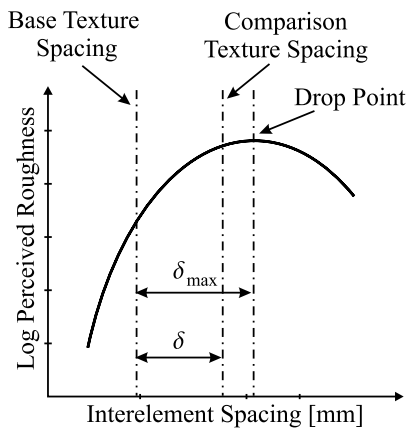

Figure 5. Psychophysical profile for roughness using dithered real textures with a probe (after [9]).

The range of BT spacings for each probe radius was selected on the basis of geometric model experiments performed in [9]. This study found a quadratic-shaped psychophysical function for roughness with element spacing. Perceived roughness was found to increase with interelement spacing up to a maximum, and then decrease. The peak of the roughness function was proposed to be related to the drop point (DP), the point at which the probe would first fall between the elements (see Fig. 5).

The JND algorithm assumes that increasing element spacing results in a rougher perceived surface, i.e., that the
CT spacing will stay on the ascending limb of the function. Thus $\delta$ will decrease as the CT spacing moves towards the BT spacing. If $\delta$ places the CT spacing on the descending limb of the function, roughness will be perceived as decreasing with increasing element spacing, violating our assumption and resulting in a "runaway" JND. This is because users would now perceive larger spacings as smoother and always answer incorrectly, resulting in greater and greater $\delta$ 's. The largest difference between CT spacing and BT spacing, $\delta_{\max }$, is therefore restricted by the DP. This sets an upper bound on the maximum BT spacing. The initial CT spacing is 1.5 times the BT. This will usually be the point of $\delta_{\max }$. Thus the maximum BT spacing for a given probe size is DP/1.5.

The DP's from [9] were used to set the upper bounds on BT spacing for probe radii ranging from $0.25-1.5 \mathrm{~mm}$. A universal minimum BT spacing of $0.5 \mathrm{~mm}$ was chosen due to device limitations. For each probe radius examined, the range from maximum to minimum BT spacing was divided into 5 equal linear steps.

\section{Experimental Results}

Each subject determined a JND for each probe radius and BT pairing. The JND determinations of a representative block for a single subject can be seen in Fig. 4. JND's for

\begin{tabular}{|c|c|c|c|c|c|}
\hline Probe Radius & \multicolumn{5}{|c|}{ Base Texture JND [mm] } \\
\cline { 2 - 6 }$[\mathrm{mm}]$ & BT1 & BT2 & BT3 & BT4 & BT5 \\
\hline 0.25 & 0.106 & 0.070 & 0.960 & 0.354 & 0.811 \\
0.50 & 0.200 & 0.126 & 0.087 & 0.114 & 0.483 \\
1.00 & 0.312 & 0.133 & 0.111 & 0.202 & 1.280 \\
1.50 & 0.474 & 0.313 & 0.190 & 0.923 & 2.348 \\
\hline
\end{tabular}

Table 2. JND's in millimeters for probe radius - base texture pairings.

each probe/BT pairing were averaged over subjects resulting in Table 2. This table is shown graphically in Figure 6. The JND's in physical units were normalized by converting them to decibel equivalents $\left(J N D_{d B}\right)$ using the formula

$$
J N D_{d B}=10 * \log _{10}((J N D+B T) / B T) .
$$

The normalized JND's of Table 3 are depicted in Figure 7.

\section{Discussion}

Looking at Figs. 6 and 7 it is immediately obvious that, for a given probe size, JND initially decreases as spacing increases. The factors that resulted in the quadratic roughness function seen in [9] explain this finding. As the probe 


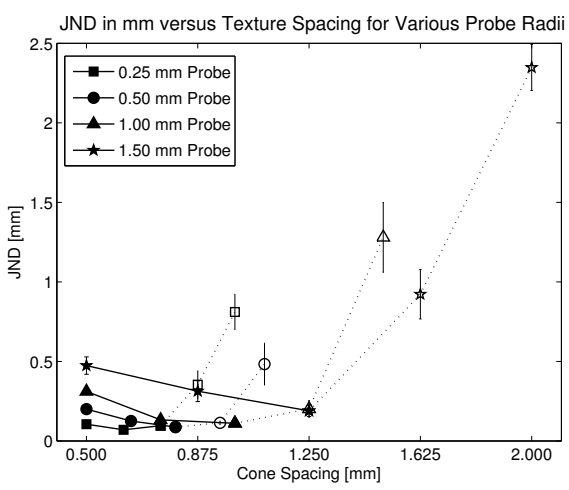

Figure 6. JND in millimeters for different probe radii over base texture spacings. Error bars indicate 1 s.e.m. JND's for the largest two BT's are dotted to indicate unreliability as discussed in the text.

moves over wider and wider spacings it approaches its DP. This is the point at which subjects feel maximum roughness since the probe is fully dropping between elements and thus moving at maximum amplitude. One would therefore expect that, close to the DP, small differences in spacing $(\delta)$ would be more noticeable than they are when far from the DP. There thus seems to be a correlation between higher subjective intensity and a greater ability to discriminate between textures. In Figs. 6 and 7 it is seen that for each probe

\begin{tabular}{|c|c|c|c|c|c|}
\hline \multirow{2}{*}{$\begin{array}{c}\text { Probe Radius } \\
{[\mathrm{mm}]}\end{array}$} & \multicolumn{5}{|c|}{ Base Texture JND [dB] } \\
\cline { 2 - 6 } & BT1 & BT2 & BT3 & BT4 & BT5 \\
\hline 0.25 & 0.808 & 0.460 & 0.512 & 1.406 & 2.530 \\
0.50 & 1.445 & 0.757 & 0.447 & 0.491 & 1.496 \\
1.00 & 2.097 & 0.698 & 0.453 & 0.634 & 2.601 \\
1.50 & 2.8542 & 1.286 & 0.605 & 1.902 & 3.358 \\
\hline
\end{tabular}

Table 3. JND's in decibels for probe radius - base texture pairings.

size, the largest two BT spacings have an increase in JND. Referring to a single subject's JND determination, seen in Fig. 4, one can see the reason. It is observed that for a 0.25 $\mathrm{mm}$ probe with a BT spacing of $1.0 \mathrm{~mm}$, the JND staircase of $\delta$ 's increases, moving away from the BT rather than approaching it. This is likely due to the quadratic shape of the psychophysical profile. For a $0.25 \mathrm{~mm}$ radius probe, the DP would be expected to be no less than $0.5 \mathrm{~mm}$, although, depending on probe speed, it might be greater. At a BT spacing of $1 \mathrm{~mm}$, spacing is well past the expected DP. The assumption that larger spacing equals rougher texture no longer holds. Instead the reverse is true and subjects perceive texture to become smoother with larger spacings. This causes the JND experiment to step in the wrong direction and run away. Trials are terminated when there have been 8 incorrect answers in a row. Thus the calculated JND's for the two largest texture spacings in each block are generally an overestimation and should not be considered accurate. The minimum JND's for probes of radius $0.25,0.5,1.0$ and

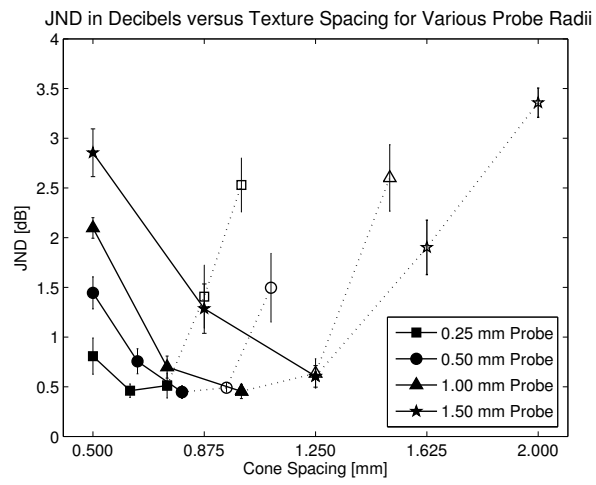

Figure 7. JND in decibels for different probe radii over base texture spacings. Error bars indicate 1 s.e.m. JND's for the largest two BT's are dotted to indicate unreliability as discussed in the text.

$1.5 \mathrm{~mm}$ are at $0.625,0.80,1.00$ and $1.25 \mathrm{~mm}$ respectively. These minima are consistently less than the psychophysically assessed magnitude maxima for real probes of similar size in [9]. For example, for a $0.5 \mathrm{~mm}$ radius probe, [9] found a maximum of $1.61 \mathrm{~mm}$ while our minimum JND occurred at $0.80 \mathrm{~mm}$. At the next measured JND (BT=0.95 $\mathrm{mm}$ ) the first CT spacing during JND determination will be $1.43 \mathrm{~mm}$. This is very close to the DP and may result in a runaway JND measurement if the subject makes an incorrect first guess.

Looking at a graph of JND's determined for varying probe radii at the same BT spacing $(0.5 \mathrm{~mm})$ it can be seen that they form a monotonic ascending series. This is explained by reference to the geometric model. At a BT spacing of $0.5 \mathrm{~mm}$, a $0.25 \mathrm{~mm}$ radius probe will drop more deeply between elements than will a $0.5 \mathrm{~mm}$ radius probe. As the probe radius increases further, the amplitude of motion as the probe passes over the elements will decrease still further. If the elements have a side angle $\alpha$ (see Fig. 1) of $90^{\circ}$ then the depth of penetration $d$ is related to probe radius $r$ and interlip spacing $s$, by [9]

$$
d=r-\sqrt{r^{2}-\left(\frac{s}{2}\right)^{2}} .
$$

Thus small changes in $s$ will have little effect on $d$ when $r$ is large but larger effects when $r$ is small. When the probe is much larger than inter-element spacing, small changes in spacing will be more difficult to detect. 


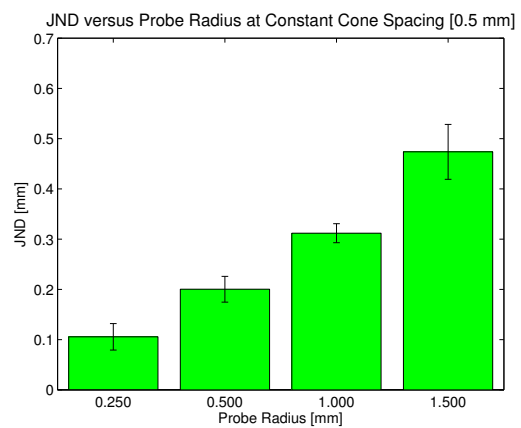

Figure 8. JND with varying probe radius on constant base texture spacing of $0.5 \mathrm{~mm}$. Error bars indicate 1 s.e.m.

\section{Conclusions and Future Work}

We have demonstrated that JND's for texture roughness can be determined using a magnetic levitation haptic device. JND experiments are simple to set up and texture variables may be quickly changed to any value along a continuum. This makes the measurement of multiple JND's an inexpensive and quick alternative to real texture JND determination.

Furthermore, we have demonstrated that JND's of texture roughness vary according to probe size and base texture spacing. JND decreases with increasing BT spacing to a minimum. JND increases with probe size for constant BT spacing. A correlation between higher subjective roughness intensity and the ability to discriminate between textures is evident. The geometric model of [9] provides an explanation for these findings.

The ease with which virtual textures can be simulated on a haptic device should allow study of a broad range of factors affecting texture perception. A logical first step would be to conduct formal validation experiments to compare real and virtual JND's. Once done, the effect of shape and positioning (i.e. dithering distance) of texture elements on texture perception could be easily investigated. Since probe force and speed may influence texture perception [9], analysis of these factors during JND determination might also be useful. One could also vary device parameters and examine the effects on JND. For example, studies show that update rate [3] and stiffness [6] may affect texture perception.

This work was supported in part by National Science Foundation grants IRI-9420869 and IIS-9802191.

\section{References}

[1] P. J. Berkelman. Tool-Based Haptic Interaction with Dynamic Physical Simulations using Lorentz Magnetic Levita- tion. PhD thesis, Carnegie Mellon University, The Robotics Institute, 1999.

[2] S. Choi and H. Z. Tan. Effect of update rate on perceived instability of virtual haptic texture. In Proceedings of the 2004 IEEE/RSJ International Conference on Intelligent Robots and Systems, volume 4, pages 3577-3582, 2004.

[3] S. Choi, L. Walker, and H. Z. Tan. Force constancy and its effect on haptic perception of virtual surfaces. ACM Transactions on Applied Perception, 2(2):89-105, 2005.

[4] J. P. Fritz and K. Barner. Stochastic models for haptic texture. In SPIE International Symposium on Intelligent Systems and Advanced Manufacturing, volume 3901, pages 3444, 1996.

[5] M. Hollins, S. J. Bensmaia, and S. Washburn. Vibrotactile adaptation impairs discrimination of fine, but not coarse, textures. Somatosensory and Motor Research, 18(4):253262, 2001.

[6] M. Hollins, F. Lorenz, A. Seeger, and R. Taylor. Factors contributing to the integration of textural qualities: Evidence from virtual surfaces. Somatosensory and Motor Research, 22(3):1-14, 2005.

[7] C. Kaernbach. Adaptive threshold estimation with unforcedchoice tasks. Perception and Psychophysics, 63(8):13771388, 2001.

[8] R. Klatzky, S. Lederman, C. Hamilton, and G. Ramsay. Perceiving roughness via a rigid probe: Effects of exploration speed. In DSC-Vol 67, Proceedings of the ASME Dynamic Systems and Control Division-1999, pages 27-33, 1999.

[9] R. L. Klatzky, S. J. Lederman, C. Hamilton, M. Grindley, and R. H. Swendsen. Feeling textures through a probe: Effects of probe and surface geometry and exploratory factors. Perception and Psychophysics, 65(4):613-631, 2003.

[10] L. E. Krueger. David Katz's der aufbau der tastwelt (the world of touch): A synopsis. Perception and Psychophysics, 7(6):337-341, 1970.

[11] S. J. Lederman. Tactile roughness of grooved surfaces: The touching process and effects of macro- and microsurface structure. Perception and Psychophysics, 16(2):385-395, 1974.

[12] M. D. R. Minsky. Computational Haptics: The Sandpaper System for Synthesizing Texture for a Force-Feedback Display. PhD thesis, Massachusetts Institute of Technology, Program in Media Arts and Sciences, 1995.

[13] D. Ruspini, K. Kolarov, and O. Khatib. Haptic interaction in virtual environments. In IROS 1997, pages 128-133, 1997.

[14] H. Schiffman. Handbook of Research Methods in Experimental Psychology, chapter 20, Psychophysics, pages 441469. Blackwell Publishing Ltd., 2003.

[15] J. Siira and D. K. Pai. Haptic texturing - a stochastic approach. In Proceedings of the 1996 IEEE International Conference on Robotics and Automation, pages 557-562, Minneapolis, Minnesota, April 1996.

[16] H. Z. Tan, B. D. Adelstein, R. Traylor, M. Kocsis, and E. D. Hirleman. Discrimination of real and virtual high-definition textured surfaces. In Symposium on Haptic Interfaces for Virtual Environment and Teleoperator Systems, pages 3-9, 2006.

[17] C. B. Zilles. Haptic rendering with the toolhandle haptic interface. Master's thesis, Massachusetts Institute of Technology, 1995. 